\title{
Economía y ética. Una revisión con base en la teoría del bienestar
}

\author{
Cristián E. Leriche Guzmán* \\ Víctor M. Sosa Godínez \\ Óscar R. Caloca Osorio
}

\begin{abstract}
Resumen: La investigación aborda el dilema que implica la relación entre economía y ética, el cual se deriva de la separación entre filosofía moral y economía positiva. Analizarlo a través de la teoría del bienestar permite acercarse a los preceptos de un cognitivismo ético o de psicología moral, mediante una propuesta distinta de individuo representativo. La finalidad es ofrecer algunos elementos para observar a la economía desde otra perspectiva, al estrechar los lazos teóricos con la ética.

Palabras clave: Economía positiva y del bienestar, Teorema de Arrow, óptimo de Pareto, ética, Homo economicus, individuo cognitivo.
\end{abstract}

\section{Economy and ethics. A revision based on the welfare theory}

Abstract: The investigation approaches the dilemma that implies the relation between economy and ethics, which derives from the separation between moral philosophy and positive economy. To analyze it through the welfare theory allows us to approach the rules of a cognitive ethics or moral psychology, by means of a different proposal of the representative individual. The purpose is to offer some elements to observe economy from another perspective, by growing closer links between theory and ethics.

Key words: Positive economy and welfare, Arrow's Theorem, optimal of Pareto, ethics, Homo economicus, cognitive individual.

Recibido: 02.06.2009

Aceptado: 02.07.2009

$* * *$

\footnotetext{
* Universidad Autónoma Metropolitana Azcapotzalco, Distrito Federal, México. E-mail: celg@correo.azc.uam.mx; sosgovic2003@yahoo.com.mx; y oscarcalo8@yahoo.com.mx. La revisión de estilo la realizó la Mtra. Karla Fernández. Se agradecen los comentarios de los lectores anónimos, estableciendo que la versión final del documento es responsabilidad sólo de los autores.
} 
El hombre sólo puede aspirar a ser feliz en tanto que se haga digno de tal felicidad, pues esa es la condición de la felicidad que la razón se exige a sí misma.

(Kant, 2002: 43)

\section{Introducción}

De manera valorativa, la felicidad es un objetivo deseable de ser alcanzado por los individuos. Sin embargo, no es la única meta que se persigue en el transitar de la vida; le acompañan otra serie de valores, como la libertad. Ésta se relaciona con la felicidad sin que sea una condición necesaria para que exista, puesto que un individuo puede perseguir la felicidad aún ante condiciones adversas, debido a que los individuos logran adaptarse y vivir disfrutando de los momentos placenteros de la vida (Sen, 1995), aun ante la persistencia de este tipo de situaciones, tal como la pérdida de libertad.

Esto remite a la idea de que se puede ser libre pero no feliz o feliz pero no libre. El considerar a la felicidad como principal objetivo nos sitúa en el contexto del utilitarismo, condición que tiene cabida en la teoría del bienestar bajo la idea de que la maximización de la utilidad del individuo lleva a la maximización por parte del colectivo de que se trate. Situación que en su planteamiento recoge las propuestas de la Ilustración, al considerar como máximo baluarte el logro de la felicidad a través del ejercicio de la razón.

Sin embargo, el esquema planteado por la economía positiva se aleja de un enfoque moral que le pudiese redituar apuntando al análisis de conductas más ricas en diversidad que en los tipos fríos y racionales (Sen, 1988: 29). Es decir, se enfoca a la relación de cierta problemática entre economía y filosofía moral, a través de una revisión de los preceptos de la teoría del bienestar y del llamado homo economicus o individuo representativo de la economía. Esto es relevante al ser la economía del bienestar el estudio de las condiciones de vida favorable o adversa que enfrentan los miembros de una sociedad en su conjunto.

En este sentido, el objetivo de la investigación es el análisis de la relación entre economía y ética visualizada a través de la teoría del bienestar, proponiendo un camino alterno ante una transformación del individuo representativo. La exposición se ha dividido en cuatro secciones. En la primera, se observa la teoría del bienestar, pasando por la agregación de las preferencias individuales y el teorema de imposibilidad de Arrow, hasta la determinación del óptimo paretiano.

En la segunda sección se aborda el planteamiento de una ética utilitarista y se hace una crítica al esquema positivo, principalmente en la manera en que éste ha dejado de lado la riqueza que brinda la relación entre 
economía y ética bajo el esquema de la teoría del bienestar. En la tercera se elabora una digresión sobre las bases de transformación de un homo economicus a un individuo cognitivo, con base en la teoría cognitiva organicista y la psicología moral de Kohlberg. La cuarta y última parte está destinada a las conclusiones.

\section{Breviario sobre la Teoría del Bienestar}

En este apartado se tratarán los problemas de agregación de funciones individuales de valor, el teorema de Arrow, la función social de bienestar, y el óptimo de Pareto. Con el propósito de destacar los elementos de contradicción y limitación del enfoque de economía positiva en su concepción de individuo representativo.

\section{El problema de la agregación}

En primer término se plantea la estructura sobre el problema de la agregación; es decir, la manera de pasar de las funciones individuales -en las cuales está incluido nuestro individuo representativo i-, a las funciones sociales. ${ }^{1}$ En este sentido, considerando una sociedad de I individuos $\mathrm{i}=1$, .... I, los cuales requieren elegir entre un conjunto de alternativas X, donde, cada individuo mantiene una valoración sobre las opciones de X, así el dilema es:

1) El diseño de una regla que valore socialmente las alternativas de $\mathrm{X}$ a partir de las valoraciones individuales sobre $\mathrm{X}$.

2) El diseño de un mecanismo de implementación que permita poner en práctica la regla diseñada.

Esto es, se requiere plantear condiciones iniciales dadas por una regla de valoración sobre las alternativas por seleccionar y se necesita que esta regla pueda ser observada en la práctica.

Estas reglas manifiestan un dominio dado por las valoraciones individuales y un co-dominio donde se presentan las valoraciones sociales. Así, la solución para este tipo de problemática se corresponde con:

a) Información que usa la regla: Las valoraciones individuales se consideran sólo como ordenamientos o como ordenamientos con intensidades consideradas; es decir, es necesario especificar el tipo y contenido informacional sobre la métrica que usa la regla.

b) Dominio de aplicación de la regla: Decidir a qué tipo de valoraciones individuales se ha de aplicar la regla: A todo posible esquema de

\footnotetext{
${ }^{1}$ Véanse (Plata, 1999) y (Sen, 1976).
} 
valoraciones individuales o sólo a un subconjunto de éstas, ya sea por selección o exclusión de las valoraciones individuales por su cuantía o cualidad informacional, que permita determinar si son relevantes o no.

c) Co-dominio de la regla o tipo de valoración social: Hay que decidir qué se entiende como valoración social. Puede ir desde un ordenamiento de los estados sociales hasta estructuras más débiles, que permitan sólo tomar decisiones cuando la sociedad se encuentre bajo un conjunto factible pero determinado de opciones.

d) Requisitos de comportamiento de la regla: Es necesario decidir sobre los juicios de valor que se quiere que satisfaga la regla. La cual puede considerarse como fuera de juicios de valor cada vez que todos los individuos están de acuerdo con ella (Sen, 1976: 78), por un mecanismo de intersubjetividad (Wellmer, 1988).

e) Mecanismo de aplicación de la regla: Para aplicar una regla diseñada hay que recabar la información que revele las características de los agentes. A priori no hay nada que nos asegure que los agentes van a revelar su valoración individual. En este caso, es conveniente diseñar métodos que permitan obtener los resultados especificados por la regla a pesar de la posible actuación estratégica de los agentes (Plata, 1999: 53 y 54).

Una manera de agregar preferencias es la regla de la mayoría. El dilema es que puede generar ciclos entre las alternativas votadas. Así una de las alternativas fue la que estableció Arrow, donde al considerar los puntos del a) al d) obtuvo un resultado de imposibilidad, pues no es posible la aplicación de todos los puntos a la vez, y de tratar de hacerlo así lo único que se obtiene es que la agregación mantiene un orden dictatorial.

\section{El teorema de imposibilidad de Arrow}

Para observar el teorema de imposibilidad de Arrow es necesario establecer condiciones iniciales para el sistema y un conjunto de definiciones. ${ }^{2}$ Donde, denotamos el conjunto de alternativas $\mathrm{X}$ asumiendo que existen $\mathrm{I}$ agentes que van de $\mathrm{i}=1, \ldots, \mathrm{I}$. Para todo agente i se tiene una relación de preferencia racional $\geq_{i}$ definida en $\mathrm{X} .{ }^{3}$ La preferencia estricta y la relación de indiferencia se derivan de $\geq_{\mathrm{i}} \mathrm{y}$ se expresan como $>_{\mathrm{i}} \mathrm{y} \sim \sim_{\mathrm{i}}$ respectivamente. Asimismo, se considera que dos alternativas no siendo distintas pueden ser indiferentes en una relación de preferencia $\geq_{i}$. Esto se requiere para poder simbolizar el conjunto de todas las posibles relaciones de preferencias racionales en $\mathrm{X}$; con la propiedad de que dos alternativas distintas no son indiferentes. Lo cual conduce a plantear los conjuntos R y P respectivamente, observando que $\mathrm{P}\ulcorner\mathrm{R}$. Esto permite establecer las siguientes defini-

\footnotetext{
${ }^{2}$ Para esta sección véanse (Mas-Collel; Whinston y Green, 1995: capítulo 21) y (Arrow, 1994).

${ }^{3}$ Es decir, se cumple con completitud y transitividad.
} 
ciones y el teorema de imposibilidad de Arrow:

Las definiciones en particular nos llevan al establecimiento de lo ya iniciado, que es la determinación existencial de una función agregadora que conduce a los individuos a una funcional de bienestar social. En este tenor, la segunda definición se establece con la finalidad de observar que la función agregadora de bienestar social cumpla socialmente con el hecho de ser eficiente en el sentido de Pareto, y en la siguiente definición se pretende establecer una selección tal que existe una condición de independencia de alternativas irrelevantes. Con ello se tiene una funcional de bienestar que es eficiente en el sentido de Pareto y que es independiente de alternativas irrelevantes; es decir, agrega las decisiones individuales en una funcional de bienestar social, elimina aquellas alternativas no relevantes para las decisiones tomadas y es necesariamente óptima.

\section{Definición 1}

Una funcional de bienestar social (o agregadora de bienestar social) se define sobre un subconjunto dado $\Lambda\left\ulcorner\mathrm{R}^{\mathrm{I}}\right.$ esta es una regla $\mathrm{F}: \Lambda \rightarrow \mathrm{R}$ que asigna una relación de preferencia racional $\mathrm{F}\left(\geq_{1}, \ldots, \geq_{\mathrm{I}}\right)$ en el dominio admisible $\Lambda\left\ulcorner\mathrm{R}^{\mathrm{I}}\right.$

\section{Definición 2}

La funcional de bienestar social F: $\Lambda \rightarrow \mathrm{R}$ es Paretiana si para todo par de alternativas $\{\mathrm{x}, \mathrm{y}\} \vdash \mathrm{X}$ y para cualquier perfil de preferencias $\left(\geq_{1}, \ldots, \geq_{I}\right) F \Lambda$, tenemos que $x$ es socialmente preferida a y, esto es $\mathrm{xF}_{\mathrm{p}}\left(\geq_{1}, \ldots, \geq_{\mathrm{I}}\right) \mathrm{y}$, cada vez que $\mathrm{x}>_{\mathrm{i}} \mathrm{y}$ para todo $\mathrm{i}$

\section{Definición 3}

Una funcional de bienestar social F: $\Lambda \rightarrow \mathrm{R}$ definida en el dominio de $\Lambda$ satisface la condición de independencia de alternativas irrelevantes sí la preferencia social entre alguna de las dos alternativas $\{\mathrm{x}, \mathrm{y}\}\ulcorner\mathrm{X}$ depende solamente de los perfiles de preferencias individuales sobre las mismas alternativas. Formalmente, para algún par de alternativas $\{\mathrm{x}, \mathrm{y}\}\left\ulcorner\mathrm{X}\right.$, y para algún par de perfiles de preferencias $\left(\mathrm{Z}_{1}, \ldots, \mathrm{Z}_{\mathrm{I}}\right) \in \Lambda \mathrm{y}$ $\left(\geq_{1}, \ldots, \geq^{\prime},{ }^{\prime}\right) F \Lambda$ con la propiedad de que para todo $\mathrm{i}, \mathrm{x} \geq_{\mathrm{i}} \mathrm{y} \sim \mathrm{x} \geq_{1}^{\prime} \mathrm{y}, \mathrm{y}$

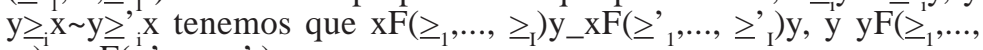
$\left.\geq_{1}\right) \mathrm{x} \geq \mathrm{yF}\left(\geq_{1}^{i}, \ldots, \geq_{1}^{\prime}\right) \mathrm{x}$

Con base en las anteriores definiciones es plausible el planteamiento del Teorema de Imposibilidad de Arrow. Sólo es necesario apuntar a ciertas condiciones: se supone que el número de alternativas es menor a tres y que el dominio de los perfiles individuales admisibles denotan $\Lambda$, sea éste cualquiera de los dos $\Lambda=\mathrm{R}^{\mathrm{I}} \mathrm{o} \Lambda=\mathrm{P}^{\mathrm{I}}$. Entonces el teorema se dicta así: cualquier funcional de bienestar social F: $\Lambda \rightarrow \mathrm{R}$ es paretiana, satisface la 
condición de independencia de alternativas irrelevantes y es dictatorial si existe un agente $h$ que para cualquier $\{x, y\} \sqcap X$ y cualquier perfil $\left(\geq_{1}, \ldots, \geq_{I}\right) \vDash \Lambda$ se tiene que $x$ es socialmente preferida a $y$, esto es que $\mathrm{xF}_{\mathrm{p}}\left(\geq_{1}, \ldots, \geq_{\mathrm{I}}\right) \mathrm{y}$ cuando $\mathrm{x} \geq_{\mathrm{h}}$ y. En este sentido, para que exista una función de bienestar social que sea agregadora, paretiana y cuente con la condición de independencia de alternativas irrelevantes, es necesario que sea dictatorial en el sentido de Arrow. ${ }^{4}$

\section{La función social de bienestar}

Planteado el Teorema de Imposibilidad, es necesario recurrir al establecimiento de la existencia de una función de bienestar social que puede ser optimizada, para lo cual, se establece un conjunto de posibilidades de utilidad como a continuación se enuncia en la siguiente definición: ${ }^{5}$

\section{Definición 4}

El conjunto de posibilidades de utilidad (CPU) es el conjunto $\mathrm{U}=\left\{\left(\mathrm{u}_{1}, \ldots, \mathrm{u}_{\mathrm{I}}\right) \neq \mathrm{R}^{\mathrm{I}}: \mathrm{u}_{1} \leq \mathrm{u}_{\mathrm{i}}(\mathrm{x}), \ldots, \mathrm{u}_{\mathrm{I}} \leq \mathrm{u}_{\mathrm{I}}(\mathrm{x})\right.$ para algún $\left.\mathrm{x} F \mathrm{X}\right\} \vdash \mathrm{R}^{\mathrm{L}}$. Donde la frontera de Pareto de $U$ se forma por los vectores de utilidad $\mathrm{u}=\left(\mathrm{u}_{1}, \ldots, \mathrm{u}_{\mathrm{I}}\right) \vDash \mathrm{U}$ para el cual no existen otras $\mathrm{u}^{\prime}=\left(\mathrm{u}_{1}, \ldots, \mathrm{u}_{\mathrm{I}}{ }_{\mathrm{I}}\right) \vDash \mathrm{U}$ que $\mathrm{u}_{\mathrm{i}}$ $\geq \mathrm{u}_{\mathrm{i}}$ para todo i y u’' $>\mathrm{u}_{\mathrm{i}}$ para algún i (véase esquema 1)

Esto describe el conjunto de restricción del constructor de política o del planeador social. Así, cualquiera de los puntos sobre la frontera de posibilidades de utilidad es un óptimo. Lo cual no deja en claro cuál es la política particular seleccionada.

\section{Esquema 1: Conjunto de posibilidades de utilidad}

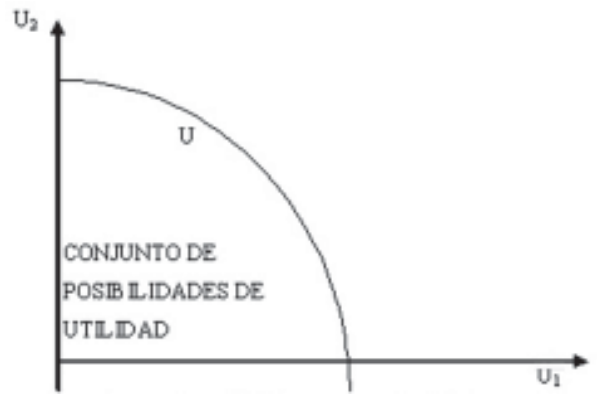

Fuente: Elaboración propia con base en figura 22.B.1 (Mas-Collel, Whinston y Green; 1995)

\footnotetext{
${ }^{4}$ Por cuestiones de espacio no mostraremos la demostración, pero ésta puede observarse en (Mas-Collel, Whinston y Green; 1995) o en (Plata, 1999). También hagamos referencia de paso en este contexto de elección social a la paradoja del liberal paretiano (Sen; 1970 y Hausman y McPherson 2007: Cap. X).

${ }^{5}$ Véase (Mas-Collel, Whinston y Green; 1995: Capítulo 22).
} 
Sin embargo, la aplicación del principio de Pareto elimina algunas políticas que conducen a los vectores de utilidad, que no se encuentran en la frontera de Pareto. Específicamente se considera que este criterio está dado por una función de bienestar social $\mathrm{W}(\mathrm{u})=\mathrm{W}\left(\mathrm{u}_{1}, \ldots, \mathrm{u}_{\mathrm{r}}\right)$ que es agregadora de las utilidades individuales en utilidades sociales. Suponemos que W(u) refleja la distribución de los juicios de valor que es la base de las decisiones del diseñador de política. Para una función de bienestar social dada $\mathrm{W}\left(\right.$.) y un conjunto de posibilidades de utilidad $\mathrm{U}$ İ $\mathrm{R}^{\mathrm{I}}$ el problema del diseñador de política es:

$$
\begin{aligned}
& \operatorname{Max} \quad W\left(\mathrm{u}_{1}, \ldots, \mathrm{u}_{\mathrm{I}}\right) \\
& \text { s.a. }\left(\mathrm{u}_{1}, \ldots, \mathrm{u}_{\mathrm{I}}\right) \rightleftharpoons \mathrm{U}
\end{aligned}
$$

Lo cual, indica la maximización de la función de bienestar social con base en las utilidades de los individuos, sujeto al conjunto de posibilidades de utilidad, en este sentido, un vector de utilidades, sobre la base de políticas determinadas, resuelve el problema, identificando a este como un óptimo social.

Con base en ello es posible determinar las propiedades de la función de bienestar social (FBS):

1. No paternalismo. La primera propiedad ya está implícita en el concepto de una FBS. Esto refleja que en la expresión de preferencias sociales únicamente las utilidades individuales cuentan: dos alternativas que son consideradas indiferentes para todo agente también. son indiferentes socialmente. El planeador no tiene preferencias directas sobre las alternativas finales. Esta es una condición en la cual un sujeto no elige una alternativa subjetivamente.

2. Propiedad Paretiana. Concede la propiedad anterior, puesto que la propiedad Paretiana es un incontroversial complemento de aquélla. Simplemente decimos que $W\left(\right.$.) es creciente donde, si u' $>{ }^{\prime} u_{i}$ para todo $i$, entonces $W\left(u^{\prime}\right) \geq W(u)$, y si $u^{\prime}>u_{i}$ para todo $i$, entonces $W\left(u^{\prime}\right)>W(u)$. Se tiene también que $W($.) es estrictamente Paretiana si ésta es estrictamente creciente; si $\mathrm{u}_{\mathrm{i}} \geq \mathrm{u}_{\mathrm{i}}$ para todo i y u' $>\mathrm{u}_{\mathrm{i}}$ para el menor i, entonces $\mathrm{W}\left(\mathrm{u}^{\prime}\right)>\mathrm{W}(\mathrm{u})$. Sí W(.) es estrictamente Paretiana, la solución de la maximización $\mathrm{W}\left(\mathrm{u}_{1}, \ldots, \mathrm{u}_{\mathrm{I}}\right)$ es necesariamente un óptimo de Pareto.

3. Simetría. La propiedad de simetría asevera que en la evaluación del bienestar social todos los agentes se encuentran sobre la misma base. Formalmente, $\mathrm{W}($.$) es simétrica si \mathrm{W}(\mathrm{u})=\mathrm{W}(\mathrm{u}$ '). Esto es, cuando la base del vector $\mathrm{u}(\mathrm{u}=(2,4,5))$ constituye una permutación de la base del vector $\mathrm{u}^{\prime}$ $\left(u^{\prime}=(4,5,2)\right)$. En otras palabras, los nombres de los agentes no son consecuencia, sólo lo son las frecuencias de los diferentes valores de utilidad.

4. Concavidad. La más importante propiedad es la concavidad de W(.). Donde la concavidad en el contexto del bienestar teórico puede ser interpretada como una aversión a la condición de desigualdad. Un camino 
sencillo nos permite ver que si $\mathrm{W}($.$) es cóncava y \mathrm{W}(\mathrm{u})=\mathrm{W}(\mathrm{u}$ '), entonces $\mathrm{W}\left(1 / 2 \mathrm{u}+1 / 2 \mathrm{u}^{\prime}\right) \geq \mathrm{W}(\mathrm{u})$ (con la desigualdad estricta sí u $\neq$ u’ y W(.), es estrictamente cóncava. Con ello se puede observar que si el conjunto de posibilidades de utilidad es convexo y simétrico, entonces el vector de utilidad que asigna algún valor de utilidad a cada agente es un óptimo social para alguna FBS simétrica y cóncava. Esto conduce al establecimiento de una solución como óptimo paretiano.

\section{La solución del óptimo como óptimo paretiano}

Es necesario tomar en consideración que la solución del problema de maximización de la función de bienestar se da por la existencia de tres aspectos: la función de transformación, la de utilidad y la de bienestar. Una situación única de óptimo económico requiere la perfecta actuación en los tres aspectos, pero el “óptimo paretiano” ha acabado por significar sólo el cumplimiento de las dos primeras, sin tener en cuenta la tercera. En consecuencia, puede darse un número infinito de óptimos paretianos, a cada uno de los cuales se les llama normalmente un óptimo, mientras el óptimo es distinguido a veces con la denominación de optimum optimorum (el mejor de los mejores) (Winch; 1975: 85).

En este sentido, es necesario que se cumplan las condiciones de eficiencia para el logro del optimum optimorum, pero no es suficiente, ya que estas condiciones se cumplen en todos los puntos de la frontera de posibilidades de utilidad, pero sólo en uno de estos puntos es tangente esta frontera al contorno de la función de bienestar. Como ya se mencionó, este corresponde con la solución al problema de maximización de la función de bienestar (véase Esquema 2).

\section{Esquema 2: Punto de bienestar máximo u optimum optimorum}

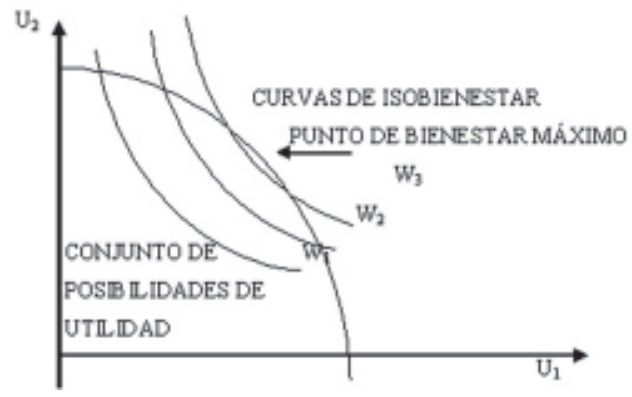

Fuente: Elaboración propia.

Así, la identificación del óptimo mejor de los mejores es una tarea que implica la concreción en políticas que permitan llevar a ese determinado estado de bienestar a la sociedad de una nación en particu- 
lar. Con ello en mente, se procede a establecer las condiciones que conducen a establecer una crítica a la economía positiva en el sentido de su desatención de la relación entre la economía del bienestar y los juicios morales.

\section{Utilitarismo y crítica a la economía positiva}

Para llevar a efecto el análisis de la relación entre economía y ética, se requiere una breve introducción al sentido que guarda el utilitarismo en el planteamiento de la economía, y cómo influye o no en la determinación de las características de la teoría del bienestar.

\section{Utilitarismo}

La mayor parte de los investigadores que refieren al utilitarismo remiten a dos pensadores de esta escuela: Jeremy Bentham y John Stuart Mill; sin embargo, cabe destacar que en muchos sentidos Mill se distanció de su maestro al comunicar que no estaba de acuerdo en diversas de las posturas que guiaban el trabajo de su mentor. (Mill, 1999 y Spiegel 1991: 436-437).

Basta observar algunas líneas al respecto, puesto que la idea "del individuo humano y del mundo por él construido como 'un conjunto de personas persiguiendo cada cual su interés o placer particular' (Mill, 1999, 71) le resulta repugnante a Mill” (Guisán, 1992: 273). John Stuart Mill no concebía al ser humano como guiado únicamente por su propio interés, como también Adam Smith no argumentaba que el individuo únicamente fuera guiado por esta condición. En términos de Sen, regularmente se ha malinterpretado a Smith por el sólo hecho de que en su obra esgrime la siguiente prosa: "No es la benevolencia del carnicero, del cervecero, o del panadero la que nos procura el alimento, sino lo consideración de su propio interés. No invocamos sus sentimientos humanitarios sino a su egoísmo, y nunca les hablamos de nuestras necesidades sino de su provecho." (Smith, 1958: 17). Este argumento debe ser complementado por la teoría de los sentimientos morales basada en la simpatía (Smith, 1997 y Rima 1995: 90-92).

Si es únicamente el egoísmo el que motiva a los seres humanos, entonces el individuo representativo de la economía positiva está limitado en facultades tales como la empatía, que necesariamente implican la interacción social. Esto remite al hecho de que la argumentación utilitarista con mayor trascendencia por sus implicaciones teóricas corresponde con la propulsada por Mill.

Ahora, si admitimos que el individuo representativo de la economía positiva está limitado para la empatía, entonces cómo suponer que podría pensar en los demás y desear el bienestar para él y para todos los demás individuos de la sociedad. Según Guisán, 
"la justificación del principio utilitarista parecería que los pasos a seguir serían los tres siguientes:

a) todo el mundo desea su felicidad (hedonismo psicológico);

b) es deseable que todo el mundo busque su felicidad (hedonismo ético egoísta);

c) es deseable que todo el mundo busque la felicidad de todo el mundo, incluida la suya propia (hedonismo ético universal)” (Guisán, 1992: 278).

Sin embargo, estos tres preceptos no se cumplen para un individuo egoísta, ya que éste desea su felicidad y no le interesa si alguien desea su felicidad. Con ello, este individuo manifiesta su desinterés en la búsqueda de la felicidad de otros. Este último punto nos aleja de la propuesta del utilitarismo, es básicamente la premisa de egoísmo ético en conjunción con el universalismo ético las que no corresponden con el individuo representativo de la economía positiva. Donde, la visión egoísta de la racionalidad

“supone, entre otras cosas, un firme rechazo de la visión de la motivación 'relacionada con la ética'. Intentar hacer lo mejor para conseguir lo que a uno le gustaría obtener puede ser una parte de la racionalidad, y esto puede incluir la promoción de objetos no egoístas que podemos valorar y desear alcanzar. Considerar la desviación de la maximización del propio interés como evidencia de la irracionalidad debe implicar un rechazo del papel de la ética en la toma real de decisiones (a menos de que se trate de alguna que otra variante de esa visión moral exótica conocida como "egoísmo ético” (Sen, 1988: 33).

Los argumentos anteriormente tratados conducen al establecimiento de las primeras bases sobre la controversia de la no inclusión de la filosofía moral en las prácticas económicas. Continuando con las observaciones sobre el utilitarismo, se tiene que el individuo representativo de la economía positiva es un sujeto que se apega más a un tipo de utilitarismo basado en el egoísmo que en el utilitarismo planteado por Mill.

Esto se refleja en el hecho de que si bien, "útil”, “utilidad”, “felicidad”, “placer”, y su consiguiente maximización no han tenido la máxima aceptación del mayor número,

“tal vez pueda deberse a malentendidos o falta de esclarecimiento, o al simple desconocimiento del significado de los términos, como ocurre con "útil” y "utilidad”, que suelen ser interpretados en el sentido pragmático común de lo que produce un determinado tipo de servicios o bienes, generalmente de tipo groseramente material, cuando el "utilitarismo" y la "utilidad”, al menos en la versión de Mil, se refieren a los bienes más preciosos y codiciados, incluida la virtud, el auto-respeto, la propia dignidad, el auto-desarrollo, etc.” (Guisán, 1992: 275). 
Esto es indicativo que la base de los preceptos del utilitarismo retomados para el individuo representativo de la economía positiva (IREP) no refieren a la valoración de Mill y sí a lo que Esperanza Guisán resalta como el "tipo groseramente material” (Guisán, 1992).

¿Por qué el IREP es del tipo grosero y no del tipo de los bienes más preciosos y codiciados? En palabras de Sen (1988: 25), esta duda puede ser esclarecida, puesto que él considera que es discutible que la importancia del enfoque ético se haya debilitado sustancialmente a medida que la economía ha evolucionado. Donde la metodología de la economía positiva no sólo ha huido del análisis normativo, sino que también ha ignorado una diversidad de complejas consideraciones éticas que afectan el comportamiento humano real.

En este sentido, el utilitarismo de un tipo y no de otro es el que sirve de telón de fondo para el planteamiento de la economía del bienestar. Ésta considera que todo individuo racional persiga su propio bienestar y que todos los individuos persigan este bienestar, pero no es del todo consistente con el hecho de que todos busquen el bienestar de todos, lo cual puede bien ser demostrado con los juegos de suma cero, donde, ante individuos racionales, lo que uno gana el otro lo pierde.

\section{Homo economicus o cómo aislar al individuo social}

En la sección anterior quedó establecido que el homo economicus o IREP no es empático y por ende carece de eficiencia en la interacción social. Esto le brinda una capacidad limitada para la generación de una personalidad moral, puesto que ésta requiere de la interacción social, el deber y la cooperación en términos de Piaget (Rubio, 1989). Si bien es cierto que éste es un constructo abstracto, también lo es el hecho de que la economía es una ciencia empírica que requiere de un individuo representativo con cualidades requeridas para la interacción social empática y por ende, para los atributos morales que le brindarían la requerida atención por parte de la economía a los juicios morales. En el ámbito macroeconómico, esto es una entidad autosustentada, mientras que en el macroeconómico no se elimina la autosustentación. Aunado a que si bien funge como parte principal de los agregados, ello no indica que exista una suerte de negociación en donde el mejor argumento detente el resultado final de la interacción. Por el contrario, al únicamente agregarse las decisiones individuales, el individuo representativo no interactúa solo, existe en un mundo de un sin número de individuos autosustentables.

Este aislamiento le confiere la particularidad de que no se interesa en los demás y es amoral. Su identidad es simple, es egoísta, guiado por su propio interés en la búsqueda de la maximización de su utilidad. Por construcción, el homo economicus en sus elecciones no integra valores que pudiesen desviar su elección, por considerar a dichos valores como una suerte de condiciones emotivas e irracionales. Este tipo de conducta atribuida al homo economicus condiciona el hecho de que se 
presenta en términos morales como un emotivista ayeriano (Sádaba, 1989). En el sentido, de considerar todo los valores morales como emotivos e irracionales y por tanto la estructuración de una teoría del bienestar que incluya diferentes valoraciones, es irracional. Esto, nos indica que el IREP se apega a los preceptos de la racionalidad y se aleja de cualesquier contenido emotivo.

Si la racionalidad del homo economicus de la teoría del bienestar es el núcleo fuerte de su sustento, entonces es necesario explorarla. ${ }^{6}$ El objetivo del IREP es, dadas unas preferencias racionales y una función de utilidad, maximizar su utilidad, pues es la condición básica que le permitirá alcanzar la felicidad.

Para ello, es necesario que cumpla con dos supuestos de racionalidad: completitud y transitividad. El primero básicamente remite a la capacidad de elección y el segundo al de racionalidad, esto es:

I. Completitud: Aquí, dadas dos canastas de políticas de bienestar, la primera es al menos tan preferida como la segunda o la segunda es al menos tan preferida como la primera. Nótese la disyunción enunciada.

II. Transitividad: Para mostrar el supuesto es necesario considerar tres canastas de políticas de bienestar: a, b, c. Se tiene que el sujeto considera a "a" tan preferible como "b" y a "b" tan preferible como "c", lo cual implica que prefiere a "a" tanto como a "c".

Es necesaria la consideración de ciertas cualidades que tienen las preferencias para la elección con base en la existencia de una utilidad. Para ello, es necesario definir los supuestos sobre las preferencias y la existencia de una función de utilidad:
1) deseabilidad,
2) monotonicidad,
3) insaciabilidad local,
4) convexidad y
5) continuidad.

Aquí se efectúa una exposición no formal puesto que es nuestro interés que la segunda parte del ensayo sea discursiva. ${ }^{7}$

Para el planteamiento del supuesto de deseabilidad se tiene que, de una manera razonable, las grandes cantidades de políticas son preferidas a pequeñas cantidades de las mismas. Tal característica de las preferencias se vincula con el supuesto de monotonicidad. Se supone que el consumo de grandes montos de bienes es siempre plausible.

\footnotetext{
${ }^{6}$ Para una revisión de las condiciones del Homo economicus véase (Zintl, 1998).

${ }^{7}$ Para una exposición formal véase (Mas-Colell et al, 1995).
} 
La monotonicidad se refiere a que, para su satisfacción, las preferencias se consideran bajo la condición de que la proporción de políticas que son un bien son mayores que las que pudiesen ser un mal. Claro, que aún con alguna política que es un mal, es posible contar con preferencias monótonas.

En este sentido, dado el supuesto de débil deseabilidad y monotonicidad, es plausible determinar un supuesto de insaciabilidad local. Ésta se corresponde con la distancia que existe entre un elemento del conjunto de consumo x y otro elemento del conjunto de consumo y, lo cual redunda en el hecho de que el bien y es estrictamente preferido al bien x, dado que tal distancia entre dichos elementos no exceda un _ determinado.

Por otra parte se plantea un supuesto de convexidad, el cual corresponde con los trade-offs que el consumidor realiza entre diferentes bienes. Con preferencias convexas se tiene que, partiendo de una situación inicial $\mathrm{x}$ de consumo, y para alguna de dos políticas consideradas, se toma cada vez un monto mayor de una de las políticas para compensar la continua perdida de unidades de la otra.

Una vez planteada la convexidad es necesario recurrir al supuesto de continuidad, para asegurar la existencia de una función de utilidad. La continuidad permite observar que las preferencias del consumidor no exhiban saltos o cortes de la función. Se considera que la relación de preferencia racional es continua.

Ahora bien, retomando el problema de elección del agente racional que nos atañe, se estipula que éste tiene una relación de preferencia que es racional, continua, convexa y localmente insaciable, y se tiene que $\mathrm{u}(\mathrm{x})$ es una función de utilidad continua representada por las preferencias. Asimismo, también se supone la existencia de un conjunto de consumo político y que el problema de elección del político es su deseo de consumir un conjunto de políticas o estrategias políticas que le permitan la maximización de su utilidad.

Están establecidas las condiciones sobre la conducta del homo economicus, el sustento básico es dado el egoísmo racional del IREP, éste maximiza su función de bienestar social encontrando el optimum optimorum. Entonces, si se encuentra un contraejemplo con el cual se sustente que la racionalidad egoísta en la búsqueda de su propio interés por parte del individuo no necesariamente conduce al mejor resultado, se tendría la posibilidad de cuestionar en sus supuestos al IREP.

El contraejemplo nos viene dado desde la teoría de los juegos, en el juego conocido como el dilema del prisionero. ${ }^{8}$ En este juego se considera la existencia de jugadores racionales y egoístas, donde cada uno de ellos

${ }^{8}$ Véase (Gibbons, 2003). 
tiene que elegir entre dos estrategias posibles: cooperar y no cooperar. En este sentido, elegirán la estrategia que les reporte la mayor utilidad posible (véase esquema 3). Los pagos se reditúan entre los jugadores según la combinación de estrategias plausibles. Para este esquema se plantean los siguientes resultados de la interacción:

Jugador A no cooperar-Jugador B no cooperar $(0,0)$

Jugador A no cooperar-Jugador B cooperar $(4,-1)$

Jugador A cooperar-Jugador B no cooperar $(-1,4)$

Jugador A cooperar-Jugador B cooperar $(3,3)$

\section{Esquema 3}

\begin{tabular}{|c|c|c|c|}
\hline & \multicolumn{2}{|c|}{ Jugador B } \\
\hline & & Cooperar & No Cooperar \\
\hline \multirow{2}{*}{ Jugador A } & Cooperar & $(3,3)$ & $(-1, \underline{4})$ \\
\hline & No Cooperar & $(\underline{4},-1)$ & $(\underline{0}, \underline{0})$ \\
\hline
\end{tabular}

Fuente: Elaboración propia.

De la interacción se observa que el jugador A, como egoísta racional, elegirá la estrategia con la utilidad más alta posible por alcanzar que es 4. En su caso, el jugador B, también como egoísta racional, elegirá la estrategia que pudiese reportarle la mayor utilidad 4. Sin embargo, al elegir estas estrategias de interacción se desprende que la situación final en la que quedan es no cooperar-no cooperar la cual les reporta una utilidad a ambos de 0 . Esto es, en la búsqueda racional de su propio interés y de la máxima utilidad posible, ambos obtuvieron perdidas, pues están en la peor situación de la interacción. Debido a que ambos ofrecen su mejor respuesta ante la mejor respuesta del otro jugador.

Lo anterior, nos indica que el argumento antes esgrimido acerca de que el IREP funciona de manera individual pero no en la interacción social, es no falso; en otras palabras, el homo economicus no busca la felicidad de todo el mundo, sólo pretende la suya, esto cuestiona el supuesto del universalismo ético. Y si esto es cuestionado, entonces, una parte del logro del bienestar social es precario, puesto que sino todos buscan el bienestar de todo el mundo, existe la alta probabilidad de encontrarnos en lo que Sen (1988: 50) plantea: “Un estado puede ser un óptimo de Pareto con algunas personas en la más grande de las miserias y con otras en el mayor de los lujos, en tanto que no se pueda mejorar la situación de los pobres sin reducir el lujo de los ricos” (Sen, 1988: 50). A continuación apuntamos más elementos acerca de la posible relación entre economía y ética. 


\section{La economía ética}

La economía en sus inicios tuvo dos orígenes distintos, que con los primeros autores se encontraban relacionados de cierta manera con las cuestiones políticas e "interesados, respectivamente, en la "ética”, por un lado, y en lo que se puede llamar la "técnica", por otro. La tradición relacionada con la ética se remonta a Aristóteles. Al comienzo de la Etica Nicomaquea, Aristóteles pone en relación la economía con los fines humanos refiriéndose a su interés por la riqueza” (Sen, 1988: 21). Estos orígenes tan diversos no proliferaron del todo, pues la técnica adquirió mayor relevancia que la conjunción con la ética, básicamente, porque los teóricos económicos tenían un fuerte problema que resolver: el tratar de mostrar que la economía era una ciencia con virtudes como las de la ciencia física, y alejarse de las acusaciones kantianas sobre preceptos metafísicos y la crítica de Comte a los conceptos metafísicos de la economía.

Si bien este distanciamiento de los preceptos éticos condujo a la economía a un rigor y fortaleza teórico-matemáticos de alto nivel, se descuidó la posibilidad de analizar los problemas económicos con un individuo representativo menos acartonado y limitado, lo cual beneficiaría en amplitud a la teoría económica y en particular a la economía del bienestar, que si bien se señalan condiciones de juicios de valor, en la realidad se piensa en elecciones racionales.

Pero en qué términos tiene que hacer su aportación la ética a la economía, en la valoración, en el sentido de que ésta tiene que guiarse por juicios morales y “adoptar una visión más amplia de lo “bueno”. De nuevo, éste es un punto que tiene alguna importancia en el contexto de la economía moderna, especialmente en la moderna economía del bienestar” (Sen, 1988: 22).

La determinación de qué es lo bueno y qué es bueno para un individuo, son condiciones no fáciles de discernir, puesto que han existido un sinnúmero de teoría éticas que han tratado de dar respuesta a estas interrogantes. Lo que si queda claro es que una ética del egoísmo, en el sentido del "egoísmo universal como realidad puede ser falso, pero el egoísmo universal como requisito de la racionalidad es evidentemente absurdo" (Sen, 1992: 33). Esta línea no es la indicada, lo necesario es apuntar sobre otra cuestión que permita incorporar lo bueno y con ello nuestros actos mentales, más que las referencias conductistas. ${ }^{9}$

Esto permitirá contar con una economía del bienestar más prístina en relación con las características tanto racionales, en sentido estricto, como las avaladas intersubjetivamente, puesto que la "situación de la economía del bienestar en la teoría económica moderna ha sido bastante precaria (...) al aumentar el recelo ante la utilización de la ética en la economía, la eco-

${ }^{9} \mathrm{Al}$ respecto véase (Pozo, 2006: capítulo 1 y 2). 
nomía del bienestar ha parecido cada día más dudosa” (Sen, 1988: 47). De hecho, se observa que difícilmente cumple con los supuestos utilitaristas mencionados con anterioridad.

El refuerzo y avance en la parte técnica redundó en una clara "opinión anti-ética, al abandonar la economía del bienestar las comparaciones interpersonales de utilidad, el criterio superviviente fue el de la optimilidad de Pareto" (Sen, 1988: 49). Sin embargo, este principio no garantiza que el beneficio sea distribuido entre los diferentes niveles de precariedad u opulencia de la sociedad de que se trate. Puesto que existe un óptimo de Pareto si y sólo si no se puede aumentar la utilidad de uno sin disminuir la utilidad de otro, una redistribución podría llevarnos a violar el óptimo pero no saca a las personas que se encuentran en una situación de precariedad de esta condición.

Además, es necesario recordar que la persistencia de una situación precaria puede llevar a los individuos a encontrar la felicidad por la voluntad de vivir, pues tanto el

“mendigo desesperado, el jornalero en situación precaria, el ama de casa dominada, el parado endurecido o el cooli exhausto pueden disfrutar de pequeñas cosas, y conseguir suprimir el intenso sufrimiento por la necesidad de continuar sobreviviendo, pero, desde el punto de vista ético sería un grave error atribuir un valor proporcionalmente reducido a la perdida de su bienestar por esta estrategia de supervivencia” (Sen, 1988: 62).

Lo anterior lleva a plantearse que existen límites en la formulación de la teoría del bienestar, cada vez que está alejada de la filosofía moral por una suerte de tecnificación de la economía que encuentra su principal fundamento amoral en su homo economicus o IREP. La nueva economía requiere un nuevo individuo representativo.

\section{Digresión sobre un nuevo homo representativo: el individuo cognitivo}

En esta sección se plantea la necesidad de un individuo representativo distinto al que corresponde a la visión de la economía positiva.

\section{Intersubjetividad y valoración}

Sin discusión, parte relevante de las acciones ejecutadas por los individuos con base en valores tienen que ver con el hecho de que, bajo las condiciones del emotivismo (Sádaba, 1989), se les atribuye un significado de subjetividad irracional. Esto en cierta medida es cierto, empero, no toda acción subjetiva necesariamente implica que es irracional, pues de ser así el individuo real no tendría espacio, a partir de su subjetividad, para ejecutar acciones que no responden con lo cánones de la racionalidad. 
Cabe destacar, que todas las acciones ejecutadas por los individuos no son objetivas, son una suerte de combinación entre acciones basadas en condiciones fundamentales, que corresponden con el apego a los datos duros del mundo físico y/o al hecho de que sólo es necesaria la coherencia lógica entre distintas elecciones, condiciones que garantizan que la conducción del individuo sea acertada, aún cuando la información sobre el evento a elegir sea incompleta; dicho de otro modo, se ejecutan elecciones en un contexto donde prolifera la incertidumbre.

El individuo ejecuta sus acciones subjetivas y obtiene o no logros, en cuyo caso, de no acertar, pudiese darse la situación de que aprendiera de sus errores y no ejecute el mismo método ante una elección similar. Esto es desalentador para los teóricos del objetivismo rampante; empero, la idea de elecciones correctas bajo circunstancias subjetivas determina que es posible considerar como objetiva una elección cada vez que ésta es considerada como correcta de manera intersubjetiva, lo cual indica que por lo menos para los especialistas en materia o los interesados en ella, la traten como objetiva.

Esto abre camino para la consideración de que los individuos conductuales tradicionales en la economía no son lo suficientemente atractivos para la incorporación de mecanismo reales a la conducta de su individuo representativo, para ello se establece un esbozo sobre el individuo cognitivo.

\section{El individuo cognitivo}

El individuo cognitivo es una estructura decisional de procesamiento de la información, misma que recibe de dos fuentes: a) por un lado, la información emanada de la experiencia y, b) por el otro, la información contenida en la memoria. El individuo cognitivo en un extremo se trata de una entidad que computa y en el otro se le considera como una identidad que posee procesos mentales, segunda condición que lo aleja del paradigma conductista. Dicho paradigma considera que existe un isomorfismo entre el contenido en el cerebro y la realidad, así que lo percibido y lo procesado de la información es el todo contenido en el cerebro.

Esta información es recolectada a través de los órganos sensoriales por medio de la percepción, la cual resulta fundamental para la estructura arquitectónica del individuo cognitivo. Pero su mayor dependencia corresponde con su necesidad de obtener y buscar información que procesar. Para expresarlo en otras palabras, en todo momento se considera que este individuo está procesando información independientemente de la percepción de un mayor o menor contenido informacional captado del mundo de la experiencia. Puesto que

"el concepto de psicología cognitiva es más amplio que el de procesamiento de la información (...) lo más general y común que podemos 
decir de la Psicología Cognitiva es que refiere la explicación de la conducta a entidades mentales, a estados, procesos y disposiciones de naturaleza mental, para los que reclama un nivel de discurso propio" (Pozo, 2006: 42).

Este supuesto de procesamiento de información es abarcativo, en el sentido de que se ejerce bajo la premisa de una descomposición recursiva de los procesos cognitivos. Esto es, cualquier hecho informativo unitario se considera complejo cada vez que puede describirse en un modo completo a un nivel más específico al descomponerse en sus hechos informativos más simples (Pozo, 2006: 45). Esto nos indica la viabilidad de observar cómo diferentes partes del cerebro procesan diferentes unidades de ese hecho informativo complejo.

A diferencia del conductismo, el paradigma del individuo cognitivo incluye las siguientes características:

- la existencia y el funcionamiento de la conciencia

- la intencionalidad de los estados mentales

- la existencia de una causación mental (Pozo, 2006: 47).

Para ello, la teoría del procesamiento de la información ha ampliado sus horizontes al lograr establecer una variante del proceso cognitivo, la cual es representada por la teoría organicistas/ estructuralistas, dentro de las cuales se parte de que la unidad de estudio de la psicología son las globalidades y que "éstas no pueden reducirse atomísticamente a los elementos que las componen (...) en la que el sujeto posee una organización propia, aunque no siempre bien definida" (Pozo, 2006: 57). Se le considera al individuo cognitivo como dispuesto y necesitado de la interacción social debido a su creciente necesidad informacional, éste toma decisiones considerando juicios morales y se apega en mayor cuantía a la realidad.

Es así como, con base en esa organización cognitiva interna del individuo, interpreta la realidad, proyectando sobre ella los significados que va construyendo. Eso conduce a un rechazo del individuo pasivo y al principio de correspondencia o isomorfismo de las representaciones con la realidad de la escuela conductista. Esto claramente indica que el individuo cognitivo es un ser activo que modifica la realidad cada vez que interactúa con ella. "Situadas en una tradición racionalista, estas teorías no creen que el conocimiento sea meramente reproductivo, sino que el sujeto modifica la realidad al conocerla (...) en el programa organicista, la actividad es inherente al sistema. El sistema no es sólo un mecanismo, es ante todo un ser vivo, un organismo" (Pozo, 2006: 57).

Estas son las características básicas del individuo cognitivo, que nos sirve de telón de fondo para reinterpretar al IREP conductista, no empático y amoral. Apuntando a el nacimiento del juicio moral. 


\section{Nacimiento de juicios morales y su relación con en el homo economicus.}

Según los resultados obtenidos por Kohlberg (Rubio, 1989: 501504) acerca del estudio realizado sobre el nacimiento del juicio moral, ${ }^{10}$ se identificaron seis estadios a diferentes niveles que evolucionan conforme lo hacen también los procesos mentales de los individuos. Para observar esto vamos a apuntar a su perspectiva social:

\section{Nivel A: Nivel preconvencional}

\section{Estadio 1: De castigo y obediencia}

Perspectiva social: este estadio adopta un punto de vista egocéntrico. Una persona en este estadio no toma en cuenta los intereses de los demás ni reconoce que difieren de los suyos; tampoco relaciona los dos puntos de vista. Las acciones las juzga en términos de consecuencias físicas más bien que en términos de intereses psicológicos de los demás. La perspectiva de la autoridad se confunde con la suya propia.

\section{Estadio 2: De designio e intercambio individual instrumental}

Perspectiva social: este estadio adopta una perspectiva individualista concreta. Una persona en este estadio separa sus intereses y sus propios puntos de vista de los de las autoridades y de los de los demás. La persona es consciente de que todos tienen intereses individuales que satisfacer y que éstos entran en conflicto, de modo que lo correcto es relativo.

\section{Nivel B. Nivel convencional}

\section{Estadio 3: De expectativas, relaciones y conformidad mutuas interpersonales}

Perspectiva social: este estadio adopta la perspectiva del individuo en relación con los demás individuos. Una persona en este estadio es consciente de los sentimientos compartidos, de los acuerdos, y de las expectativas, que tienen primacía sobre los intereses individuales. La persona relaciona los puntos de vista a través de la "Regla de Oro concreta”, poniéndose en los zapatos de los demás. No tienen en cuenta una perspectiva sistemática generalizada.

\footnotetext{
${ }^{10} \mathrm{El}$ estudio brindó sus resultados en 1986; se eligieron varones entre 10 a 17 años; se elaboró en Estados Unidos, Gran Bretaña, México, Taiwan, Belice, Bahamas, Turquía, Canadá, Israel y Nigeria.
} 


\section{Estadio 4: De mantenimiento del sistema social y de consciencia}

Perspectiva social: este estadio diferencia el punto de vista del sistema societal de los acuerdos o motivos interpersonales. Una persona en este estadio adopta el punto de vista del sistema, que define los roles y las reglas. Considera las relaciones individuales en términos de su lugar en el sistema.

\section{Nivel B/C. Nivel transicional}

Perspectiva social transicional: en este estadio la perspectiva es la de un individuo que se sitúa fuera de su propia sociedad y se considera como un sujeto que toma sus decisiones sin un compromiso general o contrato con la sociedad. Uno puede tomar y elegir obligaciones que están definidas por sociedades particulares, pero no tiene principios para tal elección.

\section{Nivel C: Nivel posconvencional y de principios}

\section{Estadio 5: De derechos prioritarios y contrato social o utilidad}

Perspectiva social: este estadio adopta una perspectiva de prioridad a la sociedad: la de un individuo racional consciente de valores y derechos prioritarios a los compromisos y contratos sociales. La persona integra las perspectivas mediante un mecanismo formal de acuerdo, contrato, imparcialidad objetiva y proceso debido. Considera el punto de vista moral y el punto de vista legal, reconoce que entran en conflicto, y encuentra difícil integrarlos.

\section{Estadio 6: De principios éticos universales}

Perspectiva social: este estadio adopta la perspectiva del punto de vista moral, del cual derivan los ordenamientos sociales, o sobre el cual se fundamentan. La perspectiva es la de todo individuo racional que reconoce la naturaleza de la moralidad o la premisa moral básica de respeto por las demás personas como fines, no como medios.

Lo anterior permite identificar en qué estadio se encuentra el homo economicus. Su estadio es el primero. Debido a cómo la economía positiva se ha distanciado de la ética, los juicios morales de su individuo representativo corresponde con los de un niño de diez años. El homo economicus es un niño egocéntrico y amoral, que no toma en cuenta los intereses de los demás, sólo los de él. Entonces, cómo suponer que puede estar operando en un universalismo ético, si en realidad este individuo no atiende a los juicios morales y no es proclive a buscar el bienestar de todo el mundo como todo el mundo pudiese estar buscando el de él. Esto nos conduce a observar que es incompatible el IREP con la inclusión de juicios morales en la teoría de bienestar. 
Esto es así, porque el IREP no es un sujeto orientado a la interacción social ni a la empatía, por lo tanto, no logra permear la ética con las condiciones en las que se encuentra el sujeto de la economía del bienestar.

Por otra parte, el individuo cognitivo tiene la facultad de transitar de los estadios menores a por lo menos el quinto, donde le permitiría a la economía enlazar su perspectiva con los juicios morales. De esta manera el individuo cognitivo puede atender de mejor manera la problemática de valor que enfrenta la teoría del bienestar. Basta recordar que este individuo requiere de la interacción social, con lo cual abre la posibilidad de pensar en los demás tanto como para procurarles que alcancen su bienestar.

\section{Conclusiones}

Apuntaremos a algunas reflexiones sobre la materia de la investigación. En primer término, si bien la teoría del bienestar busca incorporar las cuestiones valorativas del constructor de política, en realidad ésta se encuentra sujeta a los designios de un comportamiento racional, cada vez que el individuo representativo de la economía positiva es racional.

Sin embargo, esta racionalidad que se detenta y pudiese ofrecer ventajas en los designios del individuo, cuando se le somete a la práctica de la interacción social fracasa en su objetivo fundamental, que es la efectiva obtención del máximo bienestar, puesto que como se muestra en el dilema del prisionero el resultado de elección racional bajo la interacción conjunta es el peor de todos para ambos jugadores.

Así, la teoría de la elección racional no es del todo compatible con la posibilidad de que en la búsqueda del bienestar social todo mundo le interese el bienestar de todo el mundo, puesto que el mismo homo economicus, no garantiza esta premisa básica.

En segundo término se observa que el individuo representativo de la economía positiva no es empático, lo cual resulta deficiente en el momento en que éste tendría que buscar el bienestar de los demás. El egoísmo es una cuestión aún más cuestionable, puesto que el que el individuo sea egoísta y persiga su propio interés detenta el hecho de que no es altruista y por tanto, no busca cooperar con los demás, puesto que sólo vela por si y no considera los intereses de los demás.

En tercer término, al ser no empático, egoísta y alejado de obtener beneficios de la interacción social, refleja en último termino que es un individuo amoral, esto necesariamente es consecuencia del distanciamiento de la economía positiva de la ética y la falta de incorporación de los juicios morales al tipo de acción ejecutada por el homo economicus.

Si el individuo representativo es amoral, entonces ¿cómo esperar que sean viables los designios de una economía del bienestar que se susten- 
ta en las acciones de los individuos guiadas por la razón y sin apego a las normas morales? Pues simple, el homo economicus es un niño de diez años, lo cual entra en clara contradicción con uno de los preceptos de la búsqueda de la razón en Kant (2002), pues se espera que la razón saque de su minoría de edad a los hombres, pero en este caso el individuo representativo se apega a la razón pero se ubica en el primer estadio de la evolución de los juicios morales.

Es así como se propone una transformación del homo economicus en un individuo cognitivo, es decir, separar a éste de su conducta conductista y enfrentar los retos que ofrece en diversidad la teoría cognitiva, para con ello conseguir que nuevamente la economía se enlace con los preceptos de la ética con base en una concepción más amplia de la conducta humana. 


\section{Bibliografía}

Arrow, Kenneth (1994) Elección social y valores individuales, PlanetaAgostini, Buenos Aires.

Doménech, Antoni (1989) De la ética a la política, Crítica, Barcelona.

Gibbons, Robert (2003) Un primer curso de teoría de juegos, Antoni Bosch, Barcelona.

Giddens, Anthony (1995) La constitución de la sociedad. Amorrortu, Buenos Aires.

Guisán, Esperanza (1992) “Utilitarismo”, en Camps, Victoria; Guariglia, y Salmerón (eds.) Concepciones de la ética, Trotta, Madrid.

Hare, R. M. (1999) Ordenando la ética, Ariel, Barcelona.

Hausman, Daniel y M. S. McPherson (2007) El análisis económico y la filosofía moral, FCE/CIDE; México.

Kant, Immanuel (2002) Lecciones de ética, Crítica, Barcelona.

Krugman, Paul (1996) La organización espontánea de la economía, Antoni Bosch, Barcelona.

Leriche, Cristian y V. Sosa G. (1996) “El Ensayo de Robbins: una lectura necesaria”, en Leriche, C. E. (Ed.); Lecturas sobre métodos y enfoques de la economía, UAM-Azcapotzalco, México.

Mas-Colell; Andreu, Whinston; y Green (1995) Microeconomic Theory, Oxford University Press, USA.

Mill, John Stuart (1999) El utilitarismo, Alianza, Madrid.

Moreno, Rafael y C. E. Leriche (2001) "Existencia y estabilidad del equilibrio general”, en Chávez, F. (Coord.); Teoría e historia en el pensamiento económico, UAM-Azcapotzalco, México.

Plata, Leobardo (1999) “Un panorama de resultados y problemas abiertos en la teoría de la elección social”, en Barceinas, Fernando (et al); Tópicos en Economía Matemática y Econometría, UAM-Azcapotzalco, México.

Pozo, Juan (2006) Teorías cognitivas del aprendizaje, Morata, Madrid.

Ricken, Friedo (1987) Ética general, Herder, Barcelona.

Rima, Ingrid Hahne (1995) Desarrollo del análisis económico, Irwin, Madrid. 
Rubio, José (1989) “La psicología moral (De Piaget a Kohlberg)”, en: Camps, Victoria (ed.) Historia de la ética, Crítica, Barcelona.

Sádaba, Javier (1989) “Ética analítica”, en Camps, Victoria (ed.) Historia de la ética, Crítica, Barcelona.

Salcedo, Damián (1994) Elección social y desigualdad económica, Anthropos y UAM-Iztapalapa, Barcelona.

Sánchez, José Miguel y De Santiago (1998) Utilidad y Bienestar, Síntesis, Madrid.

Sen, Amartya (1970) “The imposibility of Paretian liberal”, Journal of Political Economy, vol. 78, pp. 152-157.

Idem (1976) Elección colectiva y bienestar social, Alianza, Madrid.

Idem (1988) Sobre ética y economía, Alianza, Madrid.

Idem (1995) Nuevo examen de la desigualdad, Alianza, Madrid.

Idem (2000) Desarrollo y libertad, Planeta, México.

Idem (2000) Commodities and capabilities, Oxford University Press, Nueva Delhi.

Idem (2001) El Nivel de Vida, Editorial Complutense, Madrid.

Sidgwick, Henry (1901), The methods of ethics, 6 ${ }^{\text {a }}$ Ed., Macmillan, Londres.

Smith, Adam (1997) Teoría de los sentimientos morales, Alianza Universidad, Madrid.

Idem (1957) Investigación sobre la naturaleza y causas de la riqueza de las naciones, FCE, México.

Spiegel, Henry W. (1991) El desarrollo del pensamiento económico, Omega, Barcelona.

Vilar, Gerard (1999) La razón insatisfecha, Crítica, Barcelona.

Wellmer, Albrecht (1988) “Intersubjetividad y Razón” en Olivé, León (comp.). Racionalidad, Siglo XXI, México.

Winch, D.M. (1975) Economía analítica del bienestar, Alianza, Madrid. Zintl, Reinhard (1998) Comportamiento político y elección racional, Gedisa, Barcelona. 